\title{
Research Paper \\ Effect of Classical Music on Physiological Characteristics and Observational and \\ Behavioral Measures of Pain in Unconscious Patients Admitted to Intensive \\ Care Units
}

\section{Abolfazl Rahgoi ${ }^{1}(1)$, Shahla Mohammadzadeh Zarankesh ${ }^{2}$ (1), *Sedeigheh Sadat Kasaeizadeghan ${ }^{2}\left(\mathbb{1}\right.$, Mehran Naghibeiranvand $^{3}$ (1)}

1. Department of Nursing, University of Social Welfare and Rehabilitation Science, Tehran, Iran.

2. Department of Nursing, Faculty of Nursing and Midwifery, Islamic Azad University, Tehran Medical Branch, Tehran, Iran.

3. Department of Nursing, Faculty of Nursing, Islamic Azad University, Khorram Abad Branch, Khorram Abad, Iran.

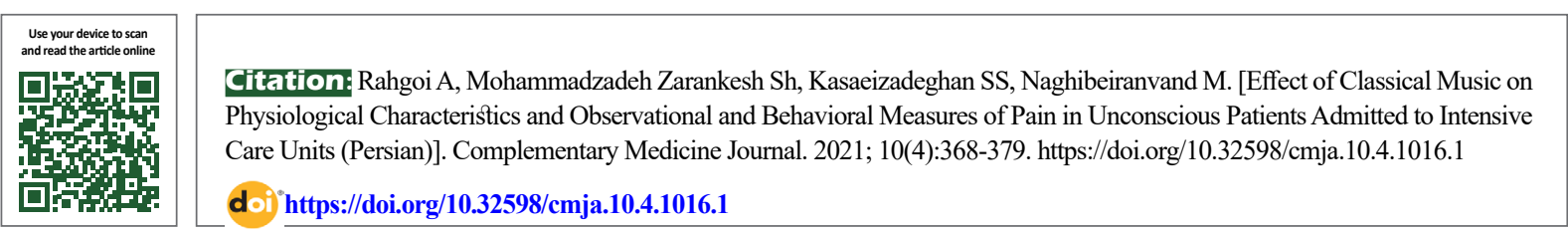

\section{(c) (i) (3)}

Article Info:

Received: 08 Sep 2020 Accepted: 15 Feb 2021 Available Online: 01 Jan 2021

Key words:

Music, Physiological characteristics, Pain, Lack of consciousness, Intensive Care Units

\section{ABSTRACT}

Objective Assessment and management of pain in patients under artificial respiration and hospitalized in Intensive Care Units (ICUs) are difficult, and is less considered by physicians and nurses. This study aims to determine the effect of classical music on physiological characteristics, and observational and behavioral measures of pain in unconscious patients admitted to ICUs.

Methods This is a quasi-experimental study conducted in 2019 on 30 unconscious patients admitted to ICU of Shohadaye Haft-e-Tir Hospital in Tehran, Iran, who were selected using a convenience sampling method, and divided into two groups of intervention and control. After obtaining a written informed consent from patients to participate, they completed a demographic/physiological form, Critical Care Pain Observation Tool (COPT) and Behavioral Pain Scale (BPC). Collected data were analyzed in SPSS V. 22 software by using descriptive and inferential statistics.

Results The Mean $\pm S D$ age of patients in the intervention and control groups was $40.33 \pm 14.01$ and $46 \pm 15.70$ years, respectively. Comparison of physiological characteristics before and after the intervention showed no statistically significant difference in any groups except in Glasgow Coma Scale score $(P<0.05)$. The mean pretest and posttest COPT scores in the intervention group were $3.73 \pm 0.94$ and $1.96 \pm 1.32$, respectively, and their mean pretest and posttest BPC scores were $5.94 \pm 1.39$ and $4.22 \pm 1.08$, respectively. Comparing the mean COPT and BPC scores before and after music therapy showed a statistically significant difference in the intervention group $(\mathrm{P}<0.05)$.

Conclusion It seems that classical music can reduce observational and behavioral measures of pain in unconscious patients hospitalized in ICUs. More studies are recommended for examining the effect of classical music on physiological characteristics of these patients.

\section{Extended Abstract}

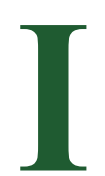

\section{Introduction}

ntensive Care Units (ICUs) are among the specialized wards of the hospital, which with its special- ized personnel and professional equipment is a suitable place for the care and treatment of critically ill patients with lifethreatening diseases. Evaluation and management of pain in patients who are under artificial respiration due to changes in the consciousness level following sedation, head trauma, or physiological status and are hospitalized in ICUs is dif-

\section{* Corresponding Author:}

Sedeigheh Sadat Kasaeizadeghan, MSc.

Address: Department of Nursing, Faculty of Nursing and Midwifery, Tehran Medical Sciences, Islamic Azad University, Tehran, Iran.

Tel: +98 (21) 55228594

E-mail: azadeh1069@gmail.com 
ficult and delayed, and it is less considered by physicians and nurses. Music therapy is a special type of psychotherapy that uses music for communication along with verbal communication. According to the World Federation of Music Therapy (WFMT), "Music therapy is the professional use of music and its elements as an intervention in medical, educational, and everyday environments with individuals, groups, families, or communities who seek to optimize their quality of life and improve their physical, social, communicative, emotional, intellectual, and spiritual health and wellbeing. Research, practice, education, and clinical training in music therapy are based on professional standards according to cultural, social, and political contexts". The current study aims to evaluate the effect of classical music on physiological characteristics, and observational and behavioral measures of pain in unconscious patients admitted to ICUs.

\section{Materials and Methods}

This is a quasi-experimental study with pretest/posttest design using a control group that was conducted in 2019 on 30 unconscious patients admitted to ICU of Shohadaye Haft-e-Tir Hospital in Tehran, Iran, who were selected using a convenience sampling method and based on the inclusion criteria. They were divided into two groups using a random number table. In addition to receiving routine care, the intervention group received classical music including the relaxing classical symphonies of Beethoven ("For Elise" for $2.56 \mathrm{~min}$ and "Violin Sonata" for $6.29 \mathrm{~min}$ ), Mozart's works ("Symphony No. 21" for 6.29 min and "Adagio in E major" for $6.45 \mathrm{~min}$ ), Bach's works for $7.36 \mathrm{~min}$ and Symphony No. 248 for $5.38 \mathrm{~min}$ (total duration= $33 \mathrm{~min}$ ), for 3 consecutive days and, then, the average score of 3 days was recorded. Pain was assessed immediately after playing the music. The songs were played to the patients in the intervention group using MP3Player and via headphones. To prevent transmission of the infection after each use, the headphones were disinfected or their pads were replaced. No music was played for the control group and only routine nursing and medical care was provided to them. Data collection tools were a demographic/physiological form, Critical Care Pain Observation Tool (COPT) and Behavioral Pain Scale (BPC).

Table 1. Comparing the physiological characteristics of patients in two groups before and after intervention

\begin{tabular}{|c|c|c|c|c|}
\hline \multirow{2}{*}{ Physiological Characteristics } & & \multicolumn{2}{|c|}{ Mean $\pm S D$} & \multirow{2}{*}{ P* } \\
\hline & & Intervention & Control & \\
\hline \multirow{3}{*}{ Pulse (beats per minute) } & Before & $16.28 \pm 96.73$ & $93.60 \pm 13.36$ & \multirow{3}{*}{$0.12<$} \\
\hline & After & $92.53 \pm 14.66$ & $95.33 \pm 14.00$ & \\
\hline & $\mathrm{P}^{* *}$ & $>0.05^{*}$ & $>0.05^{*}$ & \\
\hline \multirow{3}{*}{ Pespiration rate (breaths per minute) } & Before & $14.73 \pm 2.93$ & $15.60 \pm 2.74$ & \multirow{3}{*}{$13.0<$} \\
\hline & After & $14.46 \pm 2.94$ & $15.73 \pm 2.89$ & \\
\hline & $\mathrm{P}^{* *}$ & $>0.05^{*}$ & $>0.05^{*}$ & \\
\hline \multirow{3}{*}{ Systolic blood pressure (mm Hg) } & Before & $132.73 \pm 17.82$ & $1296.80 \pm 17.73$ & \multirow{3}{*}{$11.0<$} \\
\hline & After & $129.80 \pm 18.05$ & $127.86 \pm 16.40$ & \\
\hline & $\mathrm{P}^{* *}$ & $>0.05^{*}$ & $>0.05^{*}$ & \\
\hline \multirow{3}{*}{ Diastolic blood pressure (mm Hg) } & Before & $77.66 \pm 10.60$ & $77.73 \pm 10.12$ & \multirow{3}{*}{$15.0<$} \\
\hline & After & $79.53 \pm 17.15$ & $77.93 \pm 9.94$ & \\
\hline & $\mathrm{P} * *$ & $>0.05^{*}$ & $>0.05^{*}$ & \\
\hline \multirow{3}{*}{ Temperature $\left({ }^{\circ} \mathrm{C}\right)$} & Before & $36.92 \pm 0.25$ & $37.00 \pm 0.20$ & \multirow{3}{*}{$10.0<$} \\
\hline & After & $36.95 \pm 0.20$ & $37.07 \pm 0.46$ & \\
\hline & $\mathrm{P}^{* *}$ & $>0.05^{*}$ & $>0.05^{*}$ & \\
\hline \multirow{3}{*}{ Arterial blood oxygen saturation (percentage) } & Before & $97.60 \pm 1.72$ & $97.13 \pm 1.72$ & \multirow{3}{*}{$13.0<$} \\
\hline & After & $98.00 \pm 1.73$ & $43.1 \pm 73.97$ & \\
\hline & $\mathrm{P} * *$ & $>0.05^{*}$ & $>0.05^{*}$ & \\
\hline \multirow{3}{*}{ Glasgow Coma Scale } & Before & $8.53 \pm 8.68$ & $7.80 \pm 1.08$ & \multirow{3}{*}{$004.0>$} \\
\hline & After & $8.53 \pm 8.68$ & $7.80 \pm 1.08$ & \\
\hline & $\mathrm{P} * *$ & $>0.05^{*}$ & $>0.05^{*}$ & \\
\hline
\end{tabular}


Table 2. Comparing the mean COPT and BPC scores of patients in two groups before and after intervention

\begin{tabular}{|c|c|c|c|c|}
\hline \multicolumn{2}{|c|}{ Variables } & \multicolumn{2}{|c|}{ Mean $\pm S D$} & \multirow{2}{*}{$\mathbf{P}^{*}$} \\
\hline & & Intervention & Control & \\
\hline \multirow{3}{*}{ COPT } & Before & $3.73 \pm 0.94$ & $3.85 \pm 1.43$ & \multirow{3}{*}{$0.003>$} \\
\hline & After & $1.96 \pm 1.32$ & $4.00 \pm 1.48$ & \\
\hline & $\mathrm{P} * *$ & $<0.05$ & $>0.05$ & \\
\hline \multirow{3}{*}{ BPC } & Before & $5.94 \pm 1.39$ & $5.42 \pm 0.97$ & \multirow{3}{*}{$0.009>$} \\
\hline & After & $4.22 \pm 1.08$ & $5.56 \pm 0.93$ & \\
\hline & $\mathrm{P}^{* *}$ & $<0.05$ & $>0.05$ & \\
\hline
\end{tabular}

*Independent t-test; **Paired t-test.

Descriptive and inferential statistical tests were used to analyze the collected data in SPSS version 22 software.

\section{Results}

The Mean \pm SD age of patients in the intervention and control groups was $40.33 \pm 14.01$ and $46 \pm 15.70$ years, respectively. Comparison of their physiological characteristics before and after intervention showed that there was no statistically significant difference except in the Glasgow Coma Scale score (Table 1). Comparison of the mean scores of COPT and BPC showed a statistically significant difference in the intervention group before and after music therapy $(\mathrm{P}<0.05)$ (Table 2$)$.

\section{Conclusion}

This study showed that classical music reduces the observational and behavioral scale of pain in unconscious patients admitted to the ICU; therefore, it is recommended that nurses provide the conditions for classical music to be used as a low-cost and easy method for these patients. It is suggested that additional studies with a larger sample size using classical and preferential music be performed on unconscious patients admitted to different ICUs to examine the effect of music on physiological parameters more accurately. Nursing officials are also advised to consider this issue in the in-service training of nurses and educational planning for nursing students.

\section{Ethical Considerations}

Compliance with ethical guidelines

This study obtained its ethical approval from Islamic Azad University, Tehran Medical Branch (Code: IR.IAU.TMU.REC.1398.146). All ethical principles were observed in this study.

Funding

This study was extracted from the MA. thesis of first author at the Department of Nursing, University of Social Welfare and Rehabilitation Science, Tehran.

Authors' contributions

Conceptualization: Abolfazl Rahgavi; Data collection: Sedighe Sadat Kasaeizadegan; Data analysis: Shahla Mohammadzadeh Zarankash; Editing \& review: Mehran Naghi Beyranvand.

\section{Conflicts of interest}

The authors declareed no conflict of interest.

Acknowledgements

The authors would like to thank Islamic Azad University of Tehran Medical Branch and ICU nurses of Shohadaye Haft-e-Tir Hospital in Tehran, Iran. 


\title{
تأثير موسيقى كلاسيك بر معيارهاى فيزيولوزيك، مشاهدهاى و رفتارى درد در بيمار ان غيرهوشيار

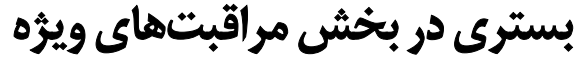

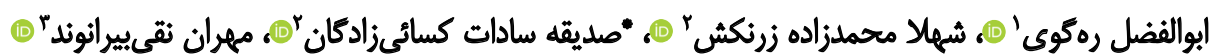

1. ا.كروه يرستاري، دانشكاه علوم بهزيستى و توانبخشى، تهران، ايران.

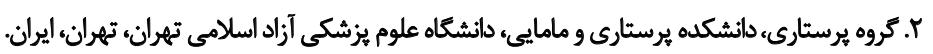

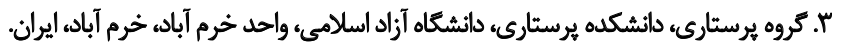

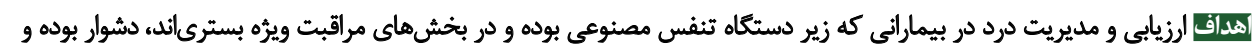

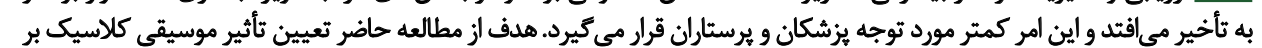

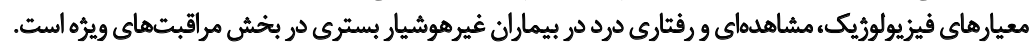

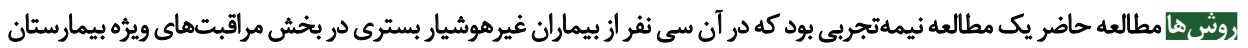

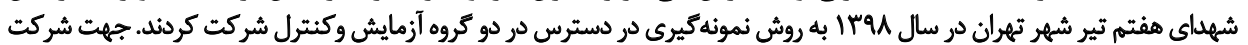

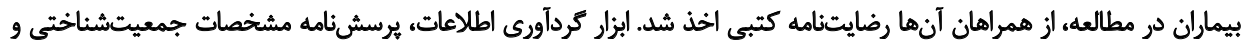

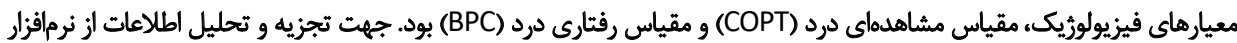
SPSS

يافته ها| ميانكين

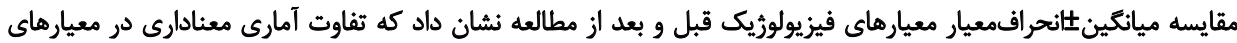

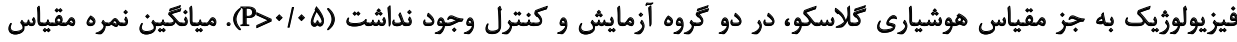

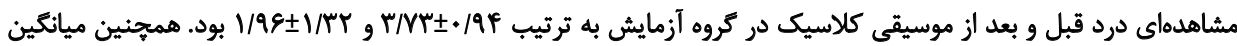

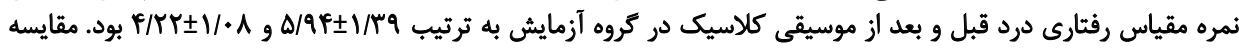

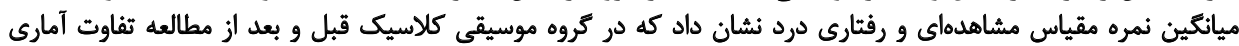

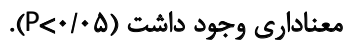

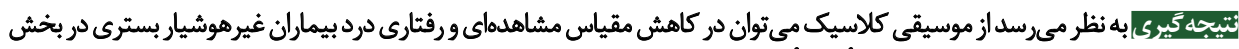

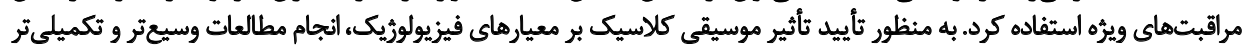

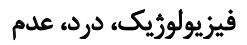

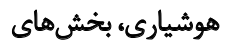
مراقبت ويره

تهاجمى، مداخلات درمانى و يرستارى و استفاده از دستكاه

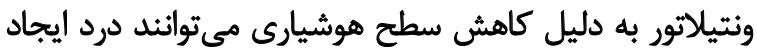

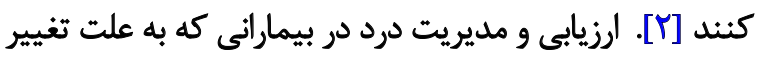

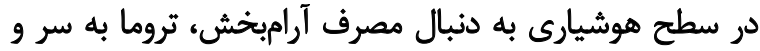

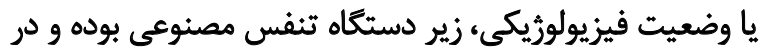

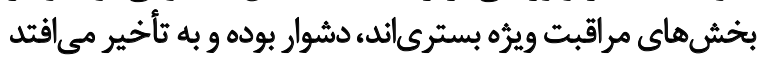

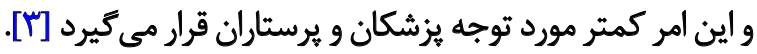
موسيقى علاوه بر اثرات هيجانى بر انسان، به طور ناخودآكاه

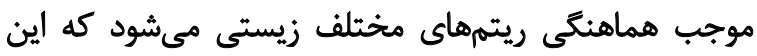

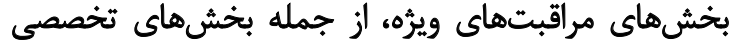

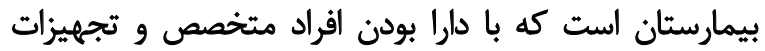

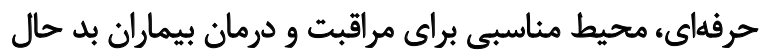

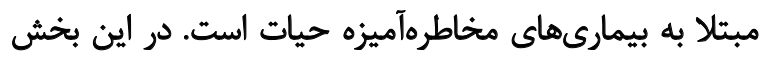

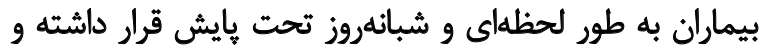

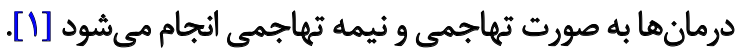
عواملى همجون بيمارىهاى حاد، جراحى، تروما، رويههاى ㅁ. 
كيفيت زندكى بيماران موثر است [•[1]. راكليو و همكاران در

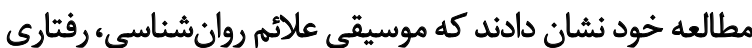

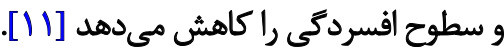

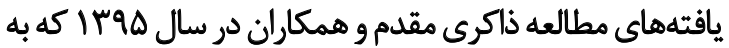

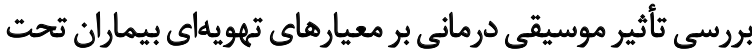

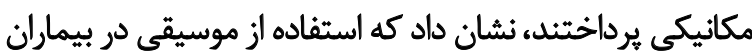

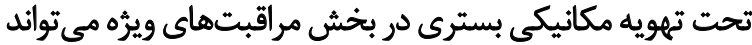

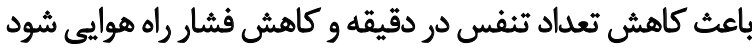

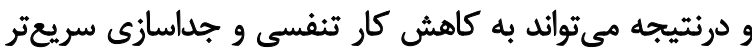

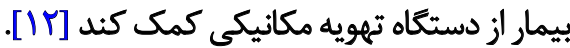

امروزه مطالعات مختلف روى بيماران مبتلا به انواع بيمارىها

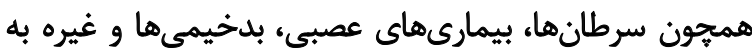

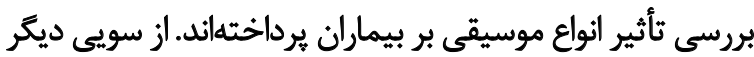

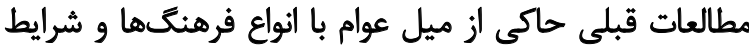

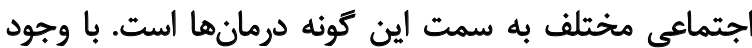

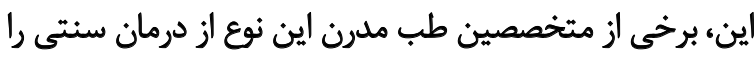
نامناسب خوانده و آن را بـ فايده مى دانند.

ضرورتى و همكاران در مطالعه خود به بررسي تأثير موسيقى

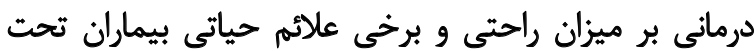

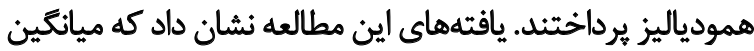

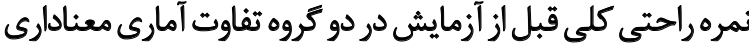

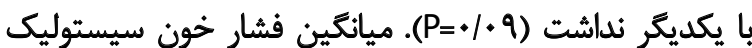

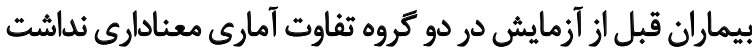

(P=•/AA)

شيروانى و همكاران مطالعهاى تحت عنوان بررسى تأثير آواى

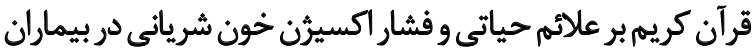

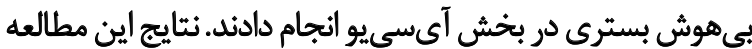

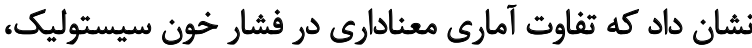

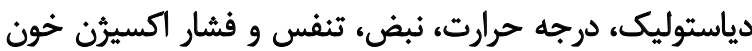

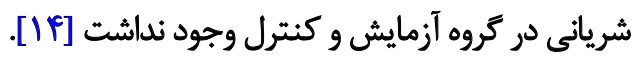

با توجه به مطالعات متناقض در مورد اثربخشى موسيقى

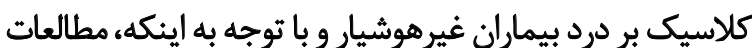

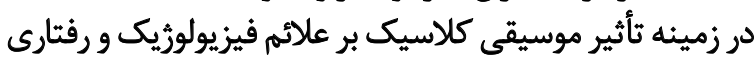

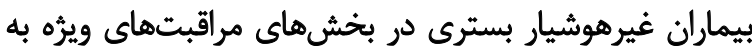
صورت بسيار محدود صورت گرفته است.

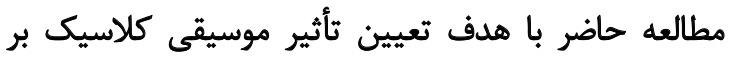

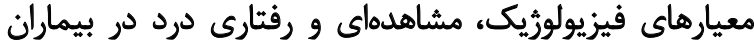

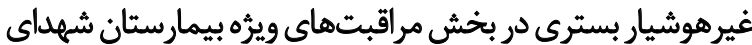

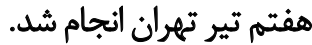

هماهنكَى بر انواع گوناكونى از حركات ارادى مانند راه رفتن، ضربه

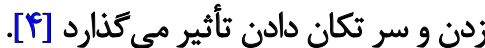

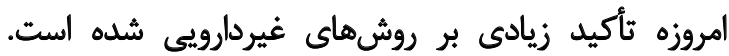

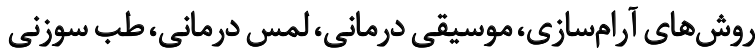

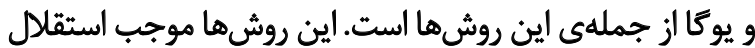

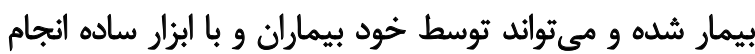

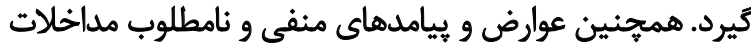
دارويى را نيز به همراه ندارند.

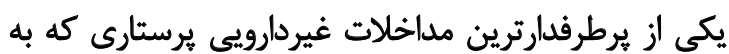

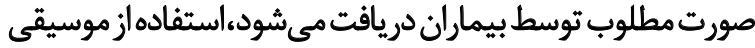

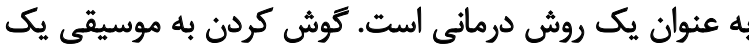

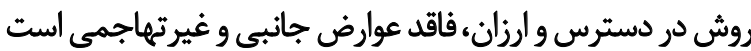

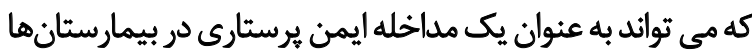

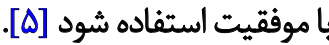

موسيقى درمانى نوع خاصى از رواندرمانى است كه در آن از از

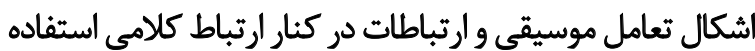

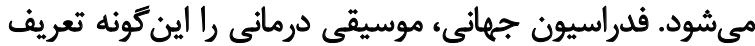

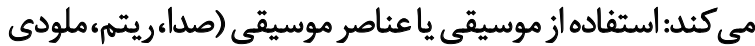

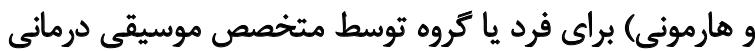

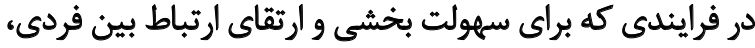

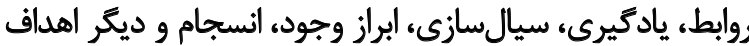

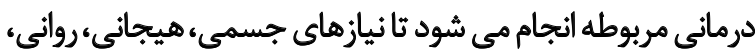

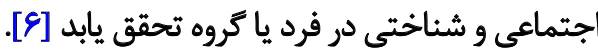
استفاده از موسيقى در شفاى بيماران ريشهى بسيار قديمى

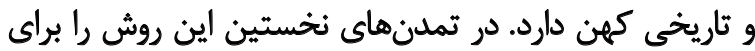

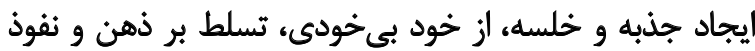

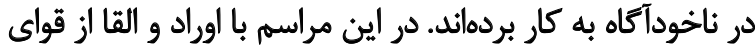

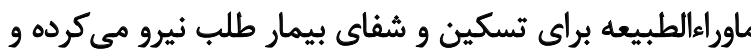
ارواح بيمارىزا را از بدن او دور مى كردانداند. در اثر ثلقين، انرثى و باورى كه در اين مراسم به وجود ميى آمد،

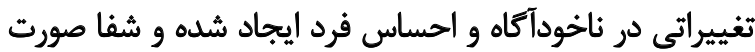

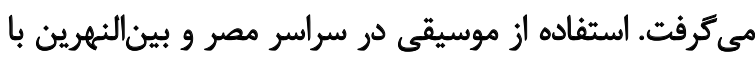

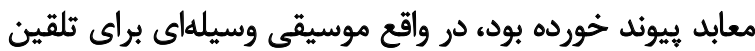

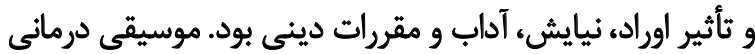

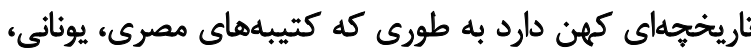

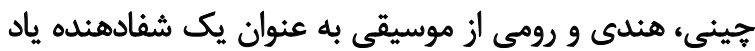

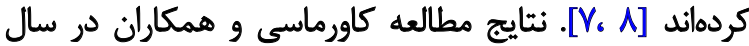

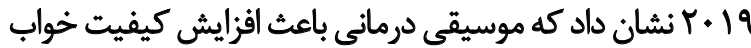

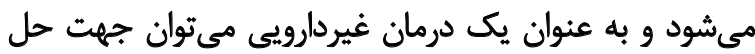

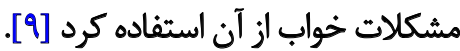

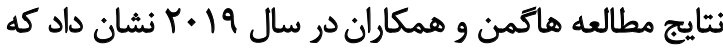

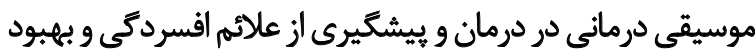


دسى كاريل تنظيم شد. جهت بيشكَيرى از انتقال عفونت ناشى از

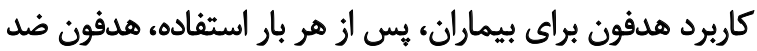

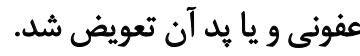

براى گروه كنترل موسيقى يخش نشد و فقيط مراقبتهاي

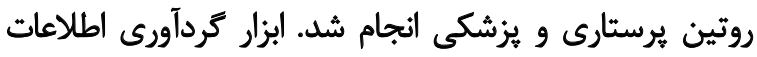

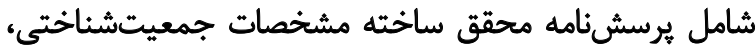

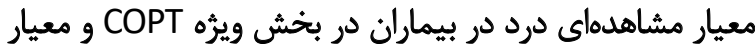

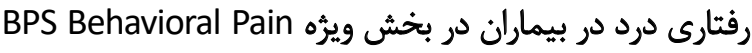
Scale مشخصات جنس، سن، علت بسترى، مدت زمان بسترى،

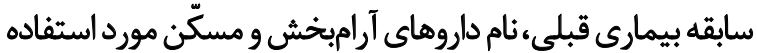

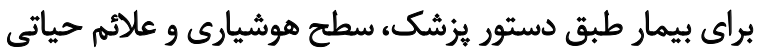

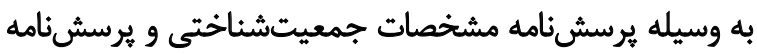

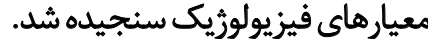

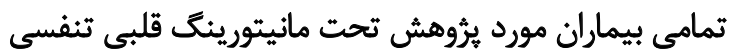

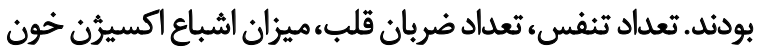

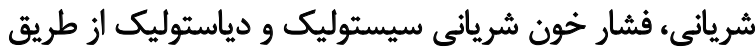

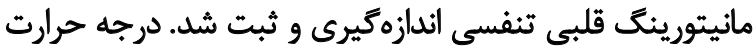

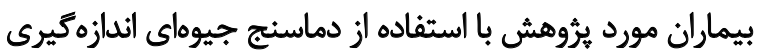

همجنين سطح هوشيارى بيماران نيز با استفاده از معيار

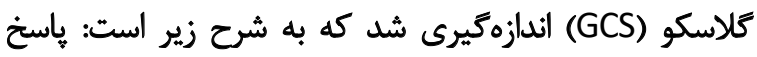

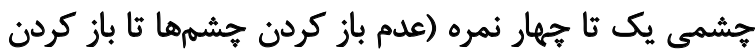

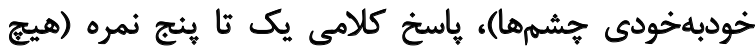

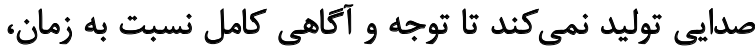

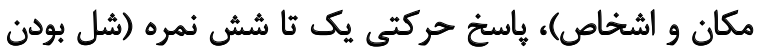

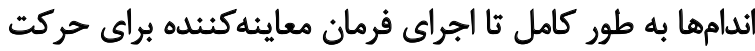
دادن قسمتهاي مختلف بدن).

معيار مشاهدهاي درد شامل جهار بخش است كه حالت جهره،

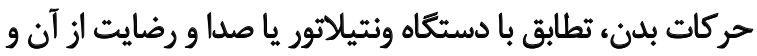

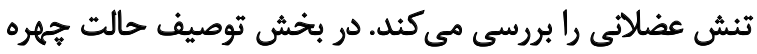

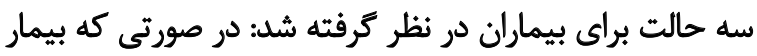

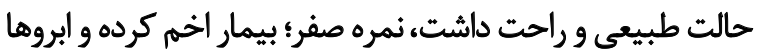

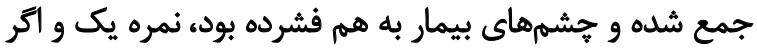

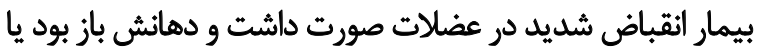
لوله تراشه داشت، نمره دو راكسب كردا

در بخش توصيف حركات بدن نيز سه حالت براى بيماران در

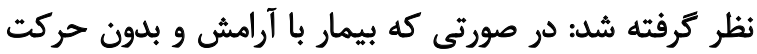

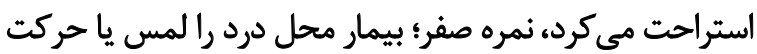

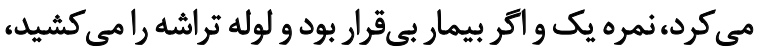

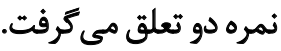

\section{مواد و روشها}

مطالعه حاضر يك مطالعه نيمهتجربى قبل و بعد با كروه كنترل

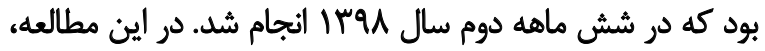

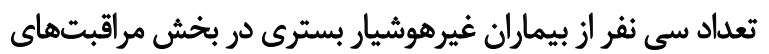

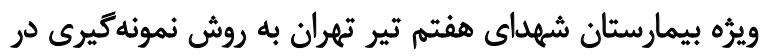

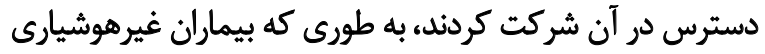

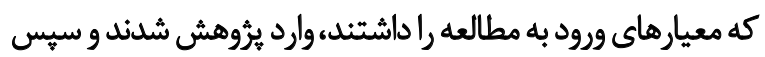

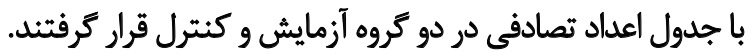

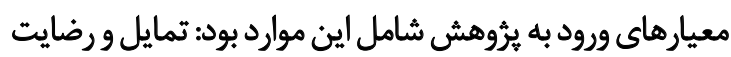

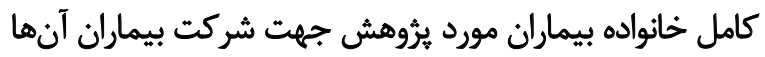

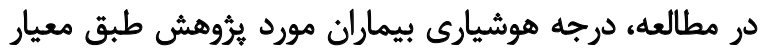

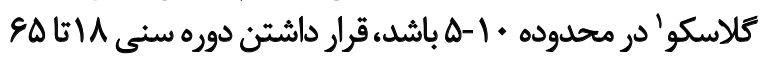

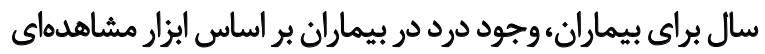

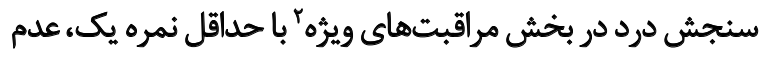

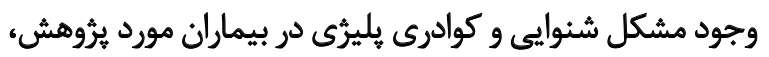

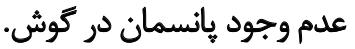

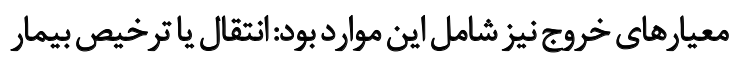

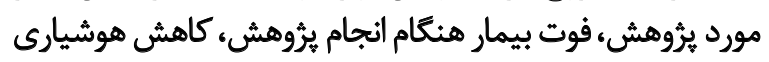

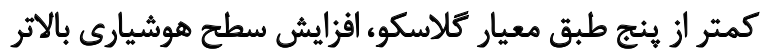

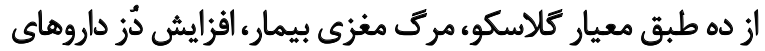

$$
\text { سداتيو و بى هوشى هئكام يثروهش. }
$$

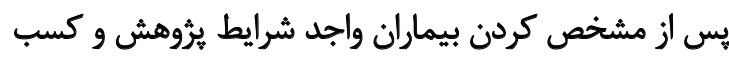

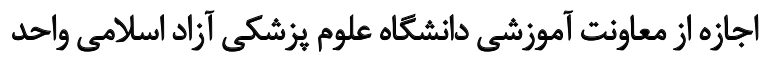

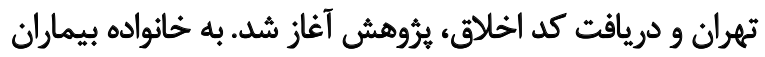

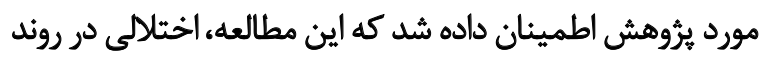

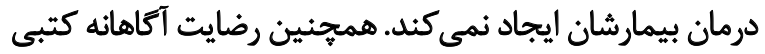

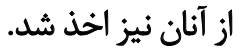

نمونهها به روش تصادفى به دو كروه پانزده نفرى تقسيم شدندي

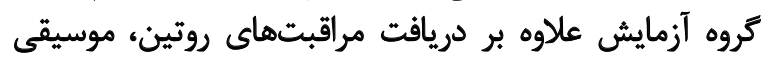

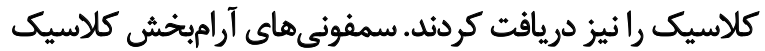

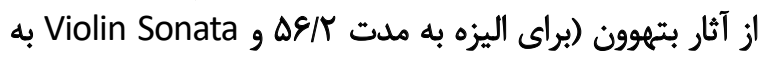

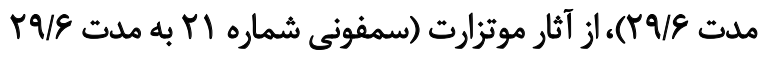

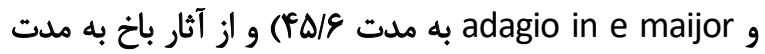
مات TrN و سمفونى TFA

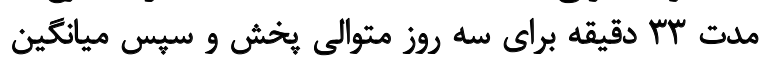

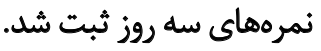

ميزان درد بلافاصله يس إز بخش موسيقى ارزيابى شد. آهنگ

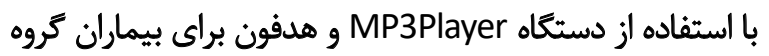

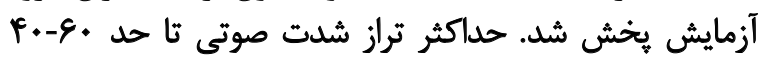

2. Critical-Care Pain Observation Tool 
به منظور تعيين روايي (اعتبار) مقياس هاى مشاهدهاي و رفتارى

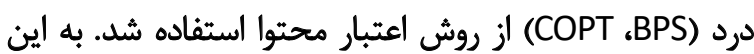

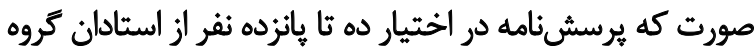

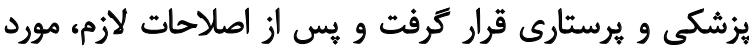

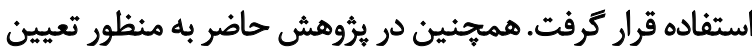

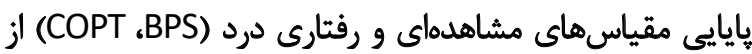

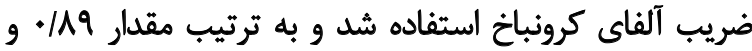

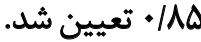

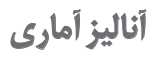

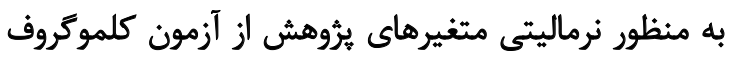

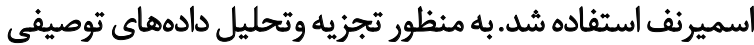

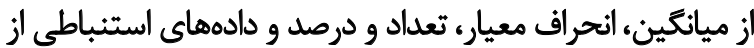

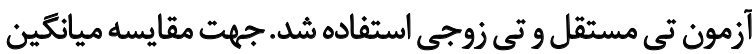

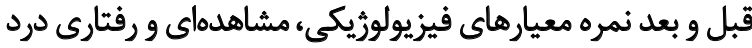
از آزمون تى زوجى استفاده شد.

همجِنين مقايسه ميانكينهاى بعد از آزمايش در دو كروه

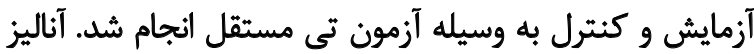

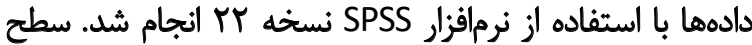

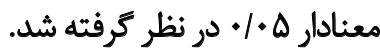

ياقتهلها

در اين مطالعه سى نفر از بيماران غيرهوشيار بسترى در بخش

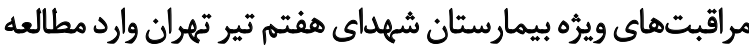

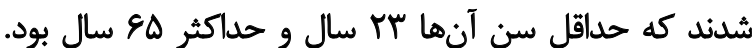

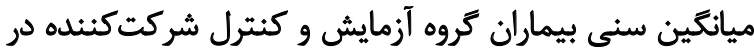

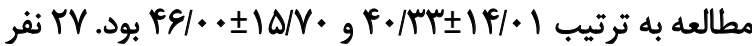

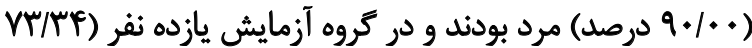

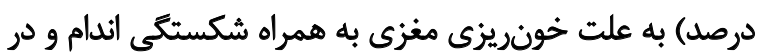

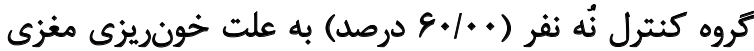

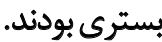

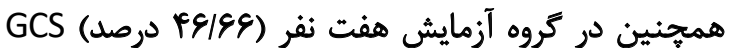

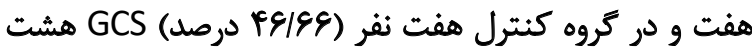

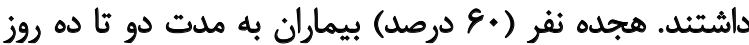

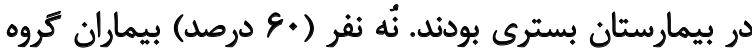

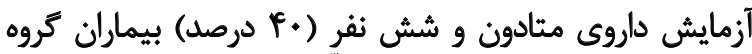

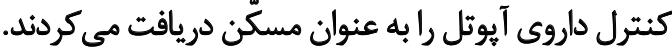
توزيع دادههاي يُروهش در هر دو كروه و در هر دو موقعيت

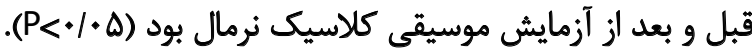

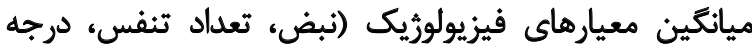

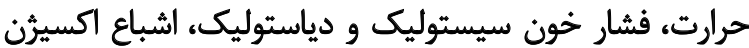

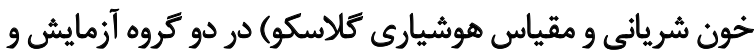

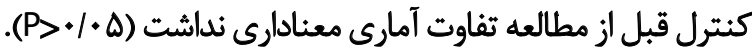

در بخش رضايت از ونتيلاتور در بيماران (اينتوبه) در صورت

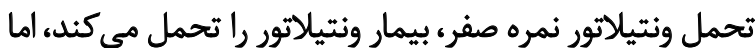

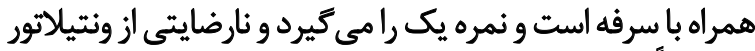

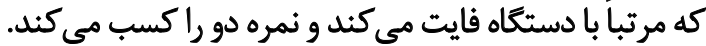
در صورتى كه بيمار "اينتوبه) نباشد، اكر بيمار بيى صدا و آرام

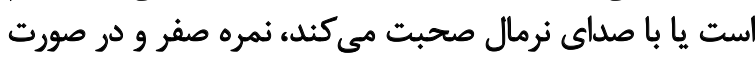

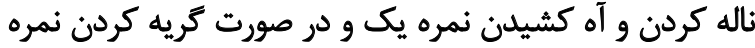

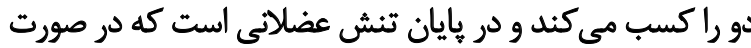

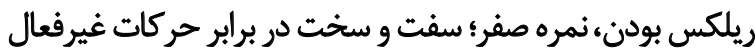

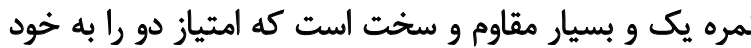

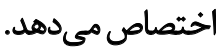

نمره كل براي هر فرد از جمع اين امتيازات به دست مى آيد كه

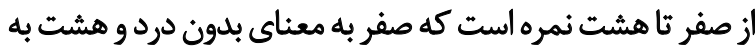

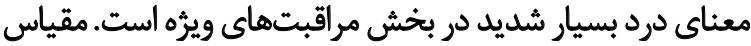

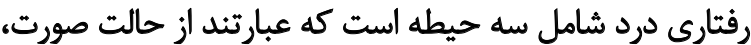
حركاث اندام فوقانى و انطباق با تهويه مكانيكى.

در بخش حالت صورت آيتمها عبارتاند از ريلكس بودن، تا

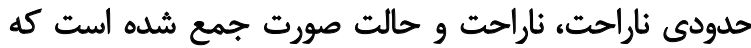
امتيازات يك تا جهار را به خود اختصاص حارت دادهاند.

در حيطه حركات اندام فوقانى آيتمها عبارتاند از بدون خماند

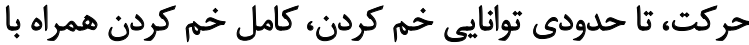

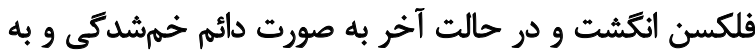

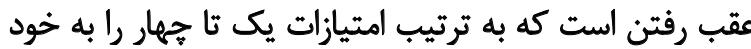

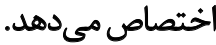

در حيطه انطباق با تهويه مكانيكى آيتمها عبارتاند از تحمل

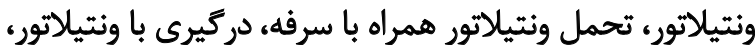

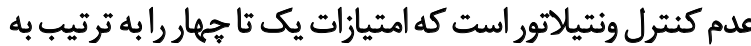

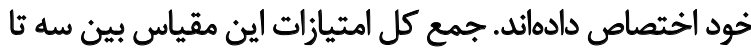

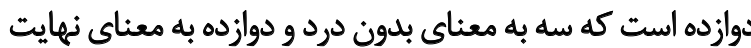

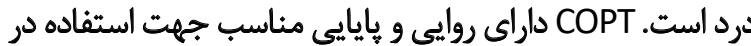

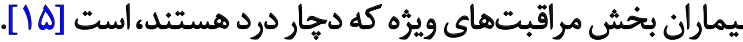
در مطالعه ذاكرى مقدم و همكاران نيز روايى و بايايى

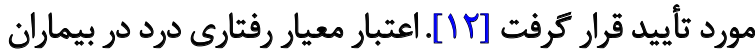

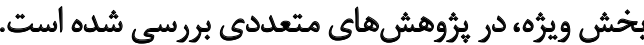
وايستت و همكاران در مطالعه خود نشان دادند كه BPS داراي

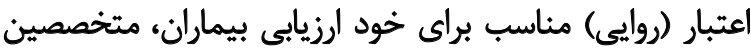

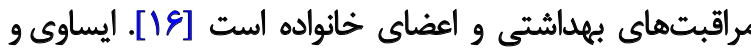

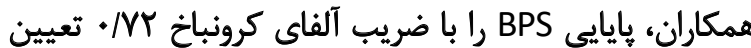

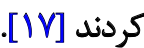

ارجى جن و همكاران نيز غايايي BPS در بيماران غيرهوشيار

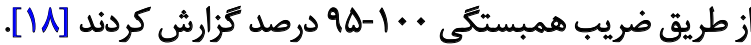


جدول ا. مقايسه ميانكين معيارهاى فيزيولوزيك بيماران غيرهوشيار مورد يروهش در كروه موسيقى كلاسيك آزمايش و كنترل و از مطالعه

\begin{tabular}{|c|c|c|c|c|}
\hline \multirow{2}{*}{ بر اساس تى مستقل از } & \multicolumn{2}{|c|}{ ميانغين土|نحراف معيار } & \multirow{2}{*}{\multicolumn{2}{|c|}{ متغيير }} \\
\hline & كتترل & 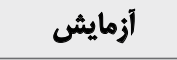 & & \\
\hline \multirow{3}{*}{$>. / M r$} & $q \Psi / 8+ \pm 1 \% / r \varepsilon$ & $q \& / M^{2} \pm 1 \& / T \wedge$ & قبيل & \multirow{3}{*}{ نبض (تعلداد در دقيقه) } \\
\hline & $q \Delta / r T \pm 1 F /$. & $9 r / \Delta r \pm I f / \& 8$ & بعد & \\
\hline & $>+1 * \Delta^{\circ}$ & $>+1.0^{\circ}$ & $\mathbf{P}$ & \\
\hline \multirow{3}{*}{$>\cdot / 1 r$} & $1 Q / 8+ \pm r / M^{f}$ & IF/ & قبل & \multirow{3}{*}{ تنفس (تعداد در دقيقه) } \\
\hline & $1 Q / R r \pm r / \wedge q$ & $\mid F / F E \pm T / q F$ & بعد & \\
\hline & $>+1 \cdot \Delta^{\circ}$ & $>+1+\Delta^{*}$ & $P$ & \\
\hline \multirow{3}{*}{$>. / 11$} & $1 r q / A+ \pm I V / N$ & IrT/ $\mathbf{r}^{\omega} \pm I V / A r$ & قبل & \multirow{3}{*}{ فشار خون سيستوليك } \\
\hline & ITV/NE $\pm \mid \& / F$. & $M Q / \Lambda \cdot \pm W \cdot \Delta$ & بعد & \\
\hline & $>+1+\infty^{*}$ & $>.1 \cdot 0^{*}$ & $\mathbf{P}$ & \\
\hline \multirow{3}{*}{$>.110$} & $W / N \pm 1 . / 1 Y$ & W/geti.le. & قبل & \multirow{3}{*}{ فشار خون دياستوليكى } \\
\hline & $W / 9 F \pm . q / 9 F$ & VQ/ATIIV/ID & بعد & \\
\hline & $>\cdot 1 \cdot 0^{*}$ & $>\cdot 1 \cdot \Delta^{*}$ & $\mathrm{P}$ & \\
\hline \multirow{3}{*}{$>.11$} & $\mathrm{~W} / \cdot \pm \pm \cdot r$ & $r E / 9 T \pm \cdot / T \Delta$ & قبل & \multirow{3}{*}{ درجه حرارت (درجه سائتى كراد) } \\
\hline & $\mathrm{Wl} \cdot \mathrm{V} \pm \cdot / \mathrm{Re}$ & $r \varepsilon / \neg \Delta \pm \cdot r$. & بعد & \\
\hline & $>+1+\infty^{\circ}$ & $>+1 \cdot \Delta^{*}$ & $\mathbf{P}$ & \\
\hline \multirow{3}{*}{$>. / 1 r$} & $9 V / I r \pm 1 / R r$ & $9 V / q \cdot \pm 1 / R$ & قبل & \multirow{3}{*}{ أشباع اكسيثن خون شريائى } \\
\hline & $9 V / V r \pm 1 / P T$ & $w \cdot . \pm 1 / N$ & بعد & \\
\hline & $>+1+\infty^{*}$ & $>.1 .0^{\circ}$ & $P$ & \\
\hline \multirow{3}{*}{$>.1 .+4$} & $V / \lambda \cdot \pm 1 / \cdot \Lambda$ & $N \Delta N^{\prime} \pm V / \& A$ & قبل & \multirow{3}{*}{ مقياس هوشيارى كالاسكو } \\
\hline & $V / A+ \pm 1 / \cdot A$ & NOrEN\&A & بعد & \\
\hline & $>+1 \cdot \infty^{\circ}$ & $>+1+\theta^{*}$ & $\mathbf{P}$ & \\
\hline
\end{tabular}

\section{ث}

نتايج اين مطالعه بيانكر آن است كه موسيقى كلاسيك نأثيرى

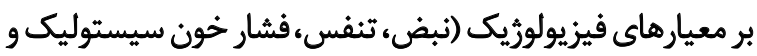

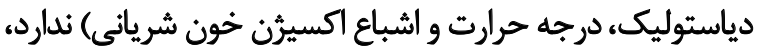

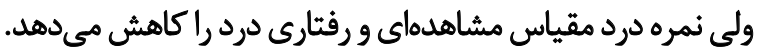

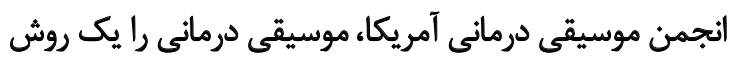

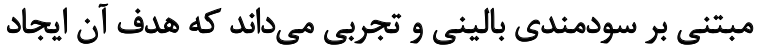

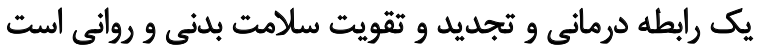

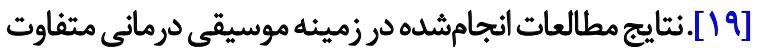

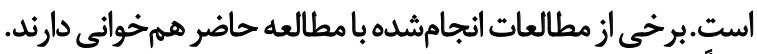

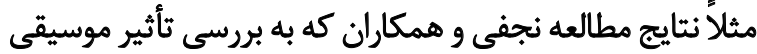

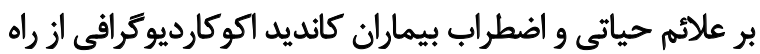
مرى يرداختئد نشان داد كه ميانكين فشارخون بيماندين سيستوليك،

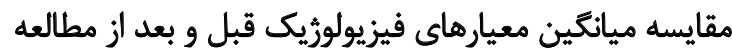

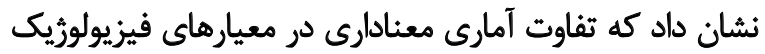

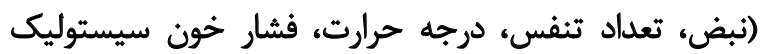

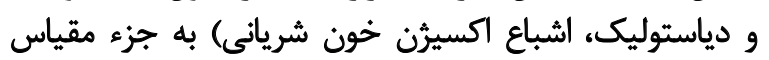

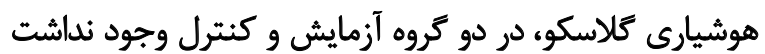

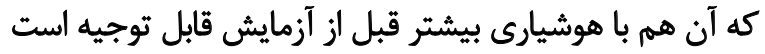

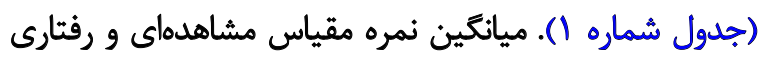

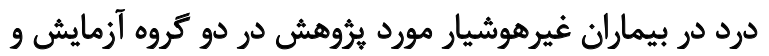

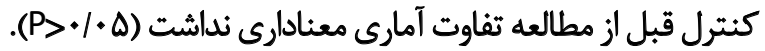

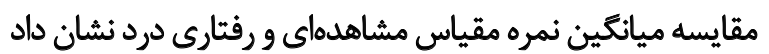

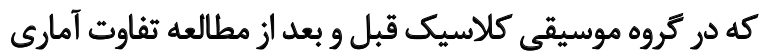

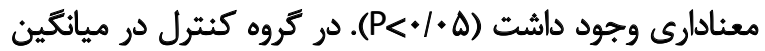

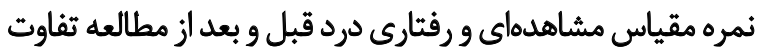

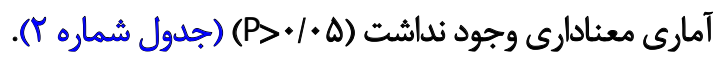


جدول r. مقايسه ميانكين نمره مقياس مشاهدهاي و رفتارى درد در بيماران غيرهوشيار مورد يثوهش در تروه موسيقى كلاسيك آزمايش وكنترل قبل و بعد از مطالعه

\begin{tabular}{|c|c|c|c|c|}
\hline \multirow{2}{*}{ بر اساس تى مستقل بعد از } & \multicolumn{2}{|c|}{ ميائكين||نحراف معيار } & & \multirow{2}{*}{ متغير } \\
\hline & كيتثرل & أزمايش & & \\
\hline \multirow{3}{*}{$<\cdot / .+r$} & $\Gamma / \Lambda \Delta \pm 1 / \Gamma$ & $r / M \Psi \pm / 9 F$ & قبل & \multirow{3}{*}{ تمره مقياس مشاهدهاي درد } \\
\hline & $f / \cdot \pm 1 / P \Lambda$ & $1 / Q \& \pm 1 / M Y$ & بعد & \\
\hline & $>.1 .0$ & $<+1 \cdot \Delta$ & $\mathbf{P}$ & \\
\hline \multirow{3}{*}{$<.1 .+9$} & $\Delta / F T \pm+/ Q V$ & $\Delta / 9 f \pm 1 / \% q$ & قبل & \multirow{3}{*}{ نمره هقياس رفتارى درد } \\
\hline & $\Delta / \Delta \& \pm . / Q T$ & $F / r r \pm 1 / \cdot A$ & بعد & \\
\hline & $>.1 .0^{\circ *}$ & $P>+1 \cdot \Delta^{\circ *}$ & $P$ & \\
\hline
\end{tabular}

مطالعات مختلفي ثأثير موسيقي بر درد بيماران بررسي كردهاند

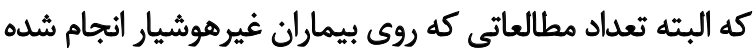

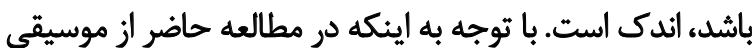

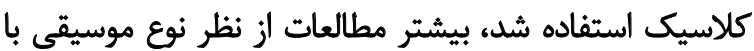

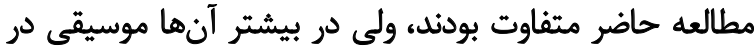
كاهش درد بيماران موثر بود.

نادرى و همكاران در مطالعه خود نشان دادند كه موسيقي

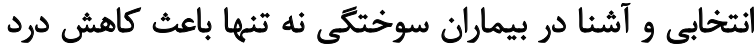

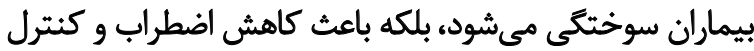

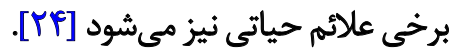
نتايج حاصل از مطالعه يعقوبينيا و همكاران كه به بررسي

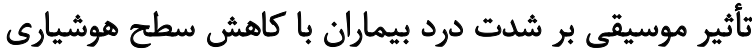

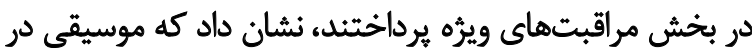

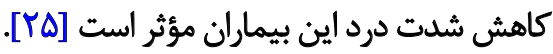
سلبي و همكاران در مطالعه خود نشان دادند كه موسيقي

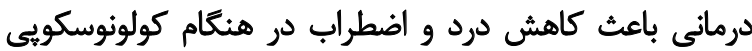

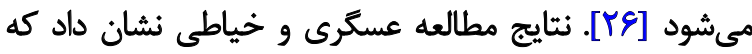

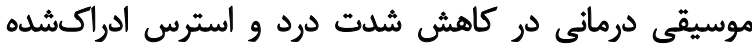
كودكان مبتلا به لوسيمي تأثير دارد [Yن] نتايج مطالعه جاسمي و همكاران كه روى بيماران سرطاني

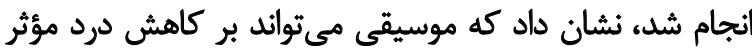

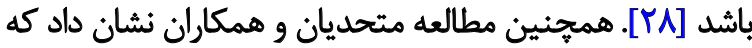

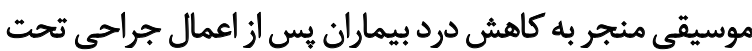

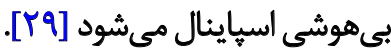

لوويس و همكاران مطالعهاي تحت عنوان تأثير موسيقي زنده

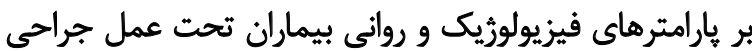

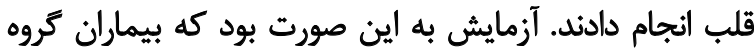

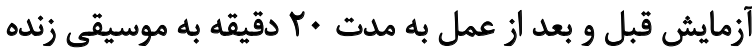

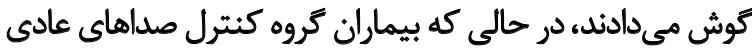

$$
\text { بيمارستان رامىشئيديند. }
$$

دياستوليك و تنفس قبل و بعد از آزمايش ثفاوت معنادارى نداشت

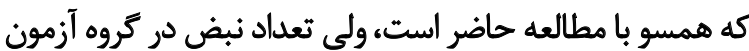
بعد از آزمايش تفاوت معنادارى داشت كه از اين نظر با مطالعا فيه

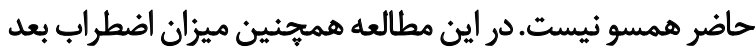

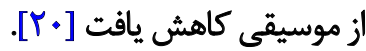

نتايج مطالعه دوآمارال و همكاران حاكي از ثأثير موسيقي بر

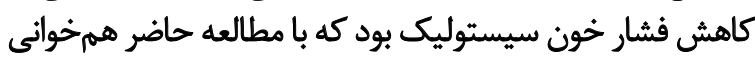

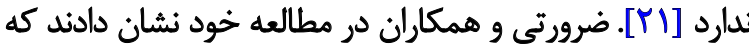

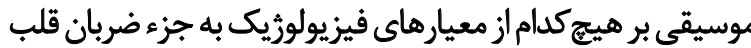
تأثير ندارد [IT] همجنين نتايج مطالعه ليو و همكاران كه به بررسي ثأثير

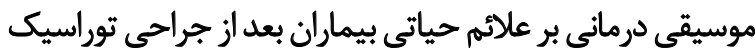

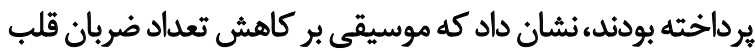

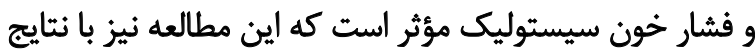

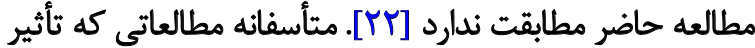
موسيقى بر بيماران غيرهوشيار را بررسى كرده باشند، اندكى بود. نتايج مطالعه ريبرو و همكاران نشان داد كه موسيقي باعث

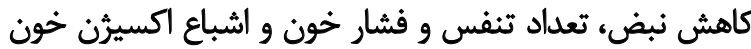

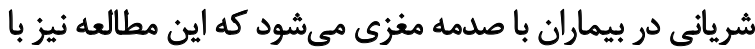
مطالعه حاضر مطابقت ندارد [بr.]. با وجود اينكه در مطالعه حاضر موسيقي هيج ثأثيرى بر ماري

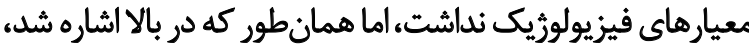

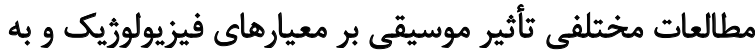

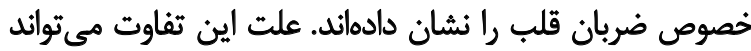

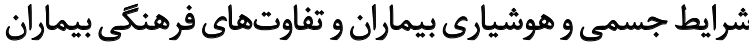

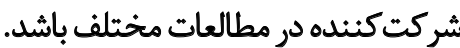
همجنين در ايران، قبلاً مطالعهاي با هدف ثأثير موسيقي

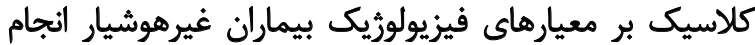

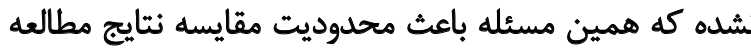
حاضر با مطالعات ديكر مى نهود. 
نكرانى خانواده بيمار در مورد اينكه آزمايش يُروهش ممكن

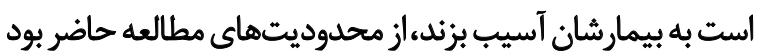

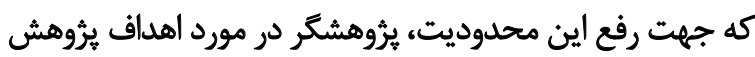

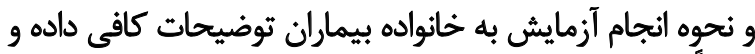

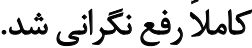

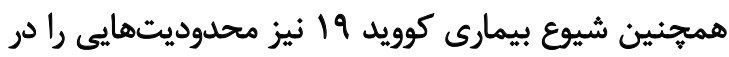

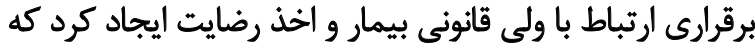

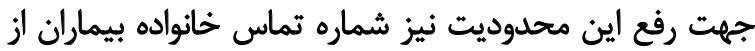

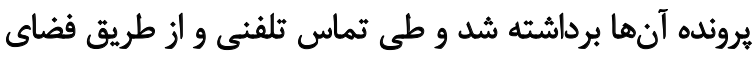

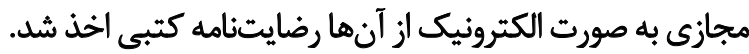

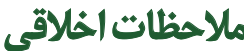

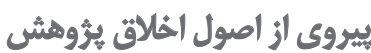

تمامى اصول اخلاق در اين تحقيق رعايت شده و مورد تاييد

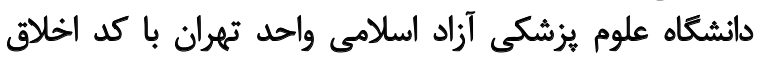

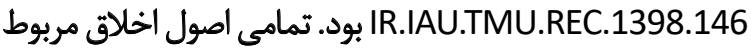
به يزوهشهاى علوم يزشكى رعايت شد.

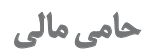

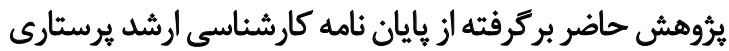

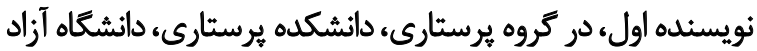
اسلامى واحد علوم يزشكى تهروان است.

مشاركت ثويسند مكان

مفهومسازى: ابوالفضل رهكوى؛ جمعآورى دادهها: صديقه

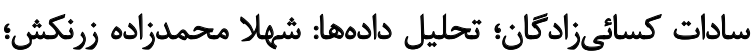

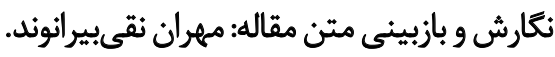

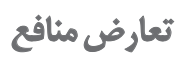

نويسندكان مفاله هيج كونه تعارضى در منافع اعلام نكردند.

$$
\text { تشكر و قدردانى }
$$

نويسندكان مراتب تقدير و تشكر خود رالز معاونت برؤوهشى دانشكاه

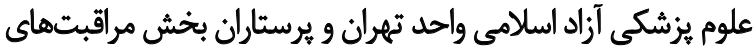

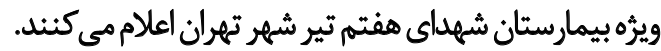

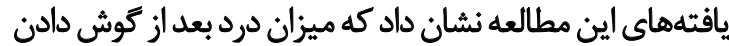

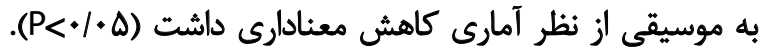

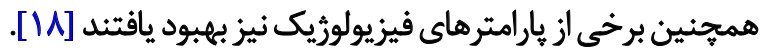

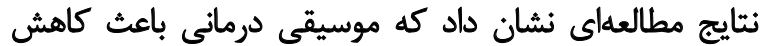

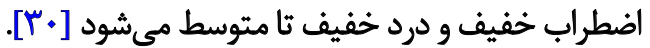
كائك ساواوراكو و همكاران مطالعهاي انجام دادند كه در آن

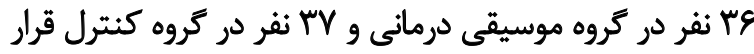

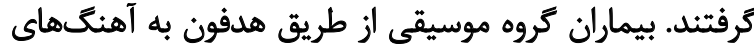

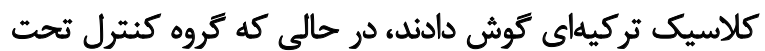

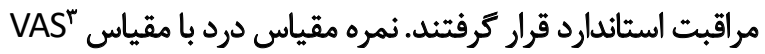
براسى شد. نتايج نشان داد كه نمره مقياس درد كروه موسيقى بعد از مداخله

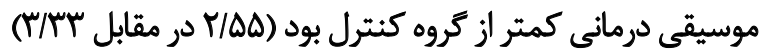

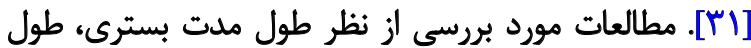

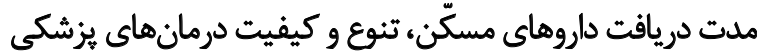

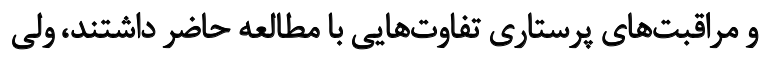

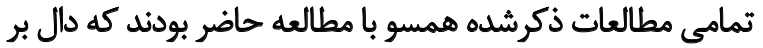
تأثير مثبت موسيقى در كنترل و كاهش درد است.

استفاده از موسيقى درمانى نه تنها عارضهاى براي بيماران

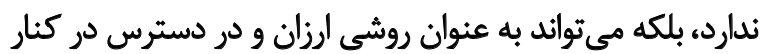

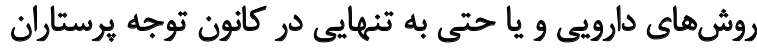

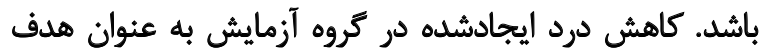

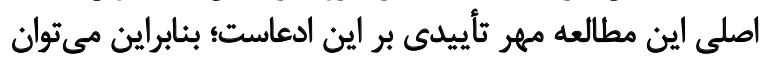

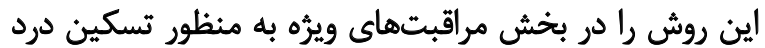

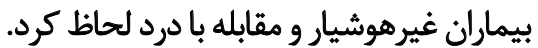

\section{نتيجلئيرى}

نتايج اين مطالعه نشان داد كه موسيقى كلاسيك منجر به

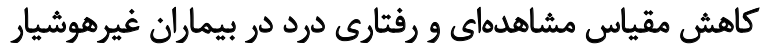
بسترى در بخش مراقبتهاى ويرٔه مي مشودي؛ بنابراين توصيه

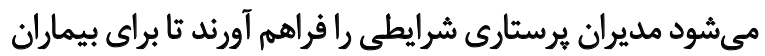

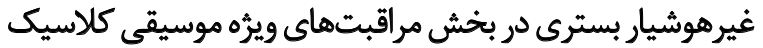
به عنوان روشى كمهزينه و آسان مورد استفاده قرار كيرد.

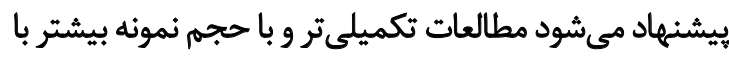

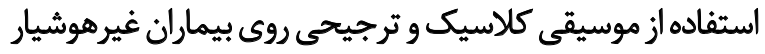

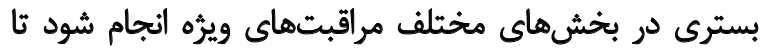

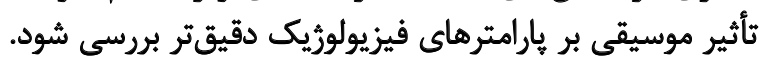

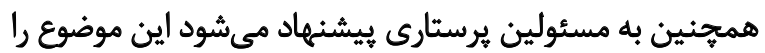

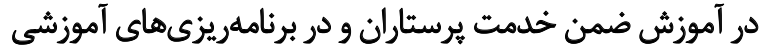
جهت دانشجويان يرستارى مورد توجه قرار دهند. 


\section{References}

[1] Marino PL. Marinos the ICU Book. Philadelphia: Lippinco Williams \& Wilkins; 2014. [DOI:10.1513/AnnalsATS.201404-164OT]

[2] Barr J, Fraser GL, Puntillo K, Ely EW, Gélinas C, Dasta JF, et al. Clinical practice guidelines for the management of pain, agitation, and delirium in adult patients in the intensive care unit. Critical Care Medicine. 2013; 41(1):263-306. [DOI:10.1097/ cCM.0b013e3182783b72] [PMID]

[3] Shahriari M, Golshan A, Ali Mohammadi N, Abbasi S. [Evaluation of the effect of a pain management program on pain control of patients by reducing the level of consciousness admitted to the intensive care units of Al-Zahra Hospital in Isfahan (Persian)]. Journal of Anesthesia and Pain. 2014; 5(1):36-45. http://jap.iums.ac.ir/ browse.php?a_id=5171\&sid=1\&slc_lang=en

[4] Bergstrom I, Seinfeld S, Arroyo J, Palacios N, Slater M, Sanchez M. V., et al. Using music as a signal for biofeedback. International Journal of Psychophysiology. 2014; 93(1):104-9. [DOI:10.1016/j. ijpsycho.2013.04.013]

[5] Han L, Li JP, Sit JW, Chung L, Jiao ZY, Ma WG. Effects of music intervention on physiological stress response and anxiety level of mechanically ventilated patients in China: A randomised controlled trial. Journal of Clinical Nursing. 2010; 19(7-8):978-87. [DOI:10.1111/j.1365-2702.2009.02845.x] [PMID]

[6] Azermai M, Petrovic M, Elseviers MM, Bourgeois J, Van Bortel LM, Vander Stichele RH. Systematic appraisal of dementia guidelines for the management of behavioural and psychological symptoms. Ageing Research Reviews. 2012; 11(1):78-86. [DOI:10.1016/j. arr.2011.07.002] [PMID]

[7] Majidi SA. [Recitation effect of holy Quran on anxiety of patients before undergoing coronary artery angiography (Persian)]. Journal of Guilan University of Medical Sciences. 2004; 13(49):61-7. https://www.sid.ir/en/journal/ViewPaper.aspx?ID=34864

[8] Ildar AE, Saleh MA, Mazloom SR. [The effect of holy quran recitation on the patient's vital signsbefore open heart surgery (Persian)]. Journal of Sabzevar University of Medical Sciences. 2003; 1(27):528. https://www.sid.ir/en/journal/ViewPaper.aspx?id=33752

[9] Kavurmaci M, Dayapoglu N, Tan M. Effect of music therapy on sleep quality. Alternative Therapies in Health and Medicine. 2019; 26(4):22-6. [PMID]

[10] Hagemann PMS, Martin LC, Neme CMB. The effect of music therapy on hemodialysis patients' quality of life and depression symptoms. Jornal Brasileiro de Nefrologia. 2019; 41(1):74-82. [DOI:10.1590/2175-8239-jbn-2018-0023] [PMID] [PMCID]

[11] Raglio A, Oasi O, Gianotti M, Manzoni V, Bolis S, Ubezio MC, et al. Effects of music therapy on psychological symptoms and heart rate variability in patients with dementia: A pilot study. Current Aging Science. 2010; 3(3):242-6. [DOI:10.2174/187460981100303 0242] [PMID]

[12] Zakeri Moghadam M, Bahadorifar H, Abbasi Z, Haghani H. [The effect of music therapy on ventilation criteria of patients under mechanical ventilation (Persian)]. Journal of Cardiovascular Nursing. 2016; 5(2):30-37. http://journal.icns.org.ir/browse.php?a $\mathrm{id}=225 \&$ sid $=1$ \&slc_lang $=$ en

[13] Zarurati M, Pishgooie SAH, Farsi Z, Karbaschi K. [The effect of music therapy on comfort level and some vital signs of patients undergoing hemodialysis (Persian)]. Military Caring Sciences Journal. 2017; 3(4):221-32. [DOI:10.18869/acadpub.mcs.3.4.221]
[14] Shirvani M, Mirzaeian R, Ghaderi A. [The effect of holy Quran sound on vital sign and Spo 2 in unconscious paents hospitalized in the ICU (Persian)]. Paper presented at: $3^{\text {th }}$ Provincial Congress on Quran and Health. 20 March 2013; Shahrekord, Iran. https://www. skums.ac.ir/page-printnew/fa/0/printskin-communique/188

[15] Hweidi IM, Nizamli FM. Stressors in intensive care units in Syria: Patients perceptions. Journal of Research in Nursing. 2015; 20(2):114-26. [DOI:10.1177/1744987114524202]

[16] Vincent JL, Shehabi Y, Walsh TS, Pandharipande PP, Ball JA, Spronk P et al. Comfort and patient-centred care without excessive sedation: The eCASH concept. Intensive Care Medicine. 2016; 42(6):962-71. [DOI:10.1007/s00134-016-4297-4] [PMID] [PMCID]

[17] Aissaoui Y, Zeggwagh AA, Zekraoui A, Abidi K, Abouqal R. Validation of a behavioral pain scale in critically ill, sedated, and mechanically ventilated patients. Anesthesia and Analgesia. 2005; 101(5):1470-6 [DOI:10.1213/01.ANE.0000182331.68722.FF] [PMID]

[18] Chen J, Lu Q, Wu XY, An YZ, Zhan YC, Zhang HY. Reliability and validity of the Chinese version of the behavioral pain scale in intubated and non-intubated critically ill patients: Two cross-sectional studies. International Journal of Nursing Studies. 2016; 61:63-71. [DOI:10.1016/j.jinurstu.2016.05.013] [PMID]

[19] Debra S, Susan M, Yan T, Russell E, Larry D. Music therapy is associated with family perception of more spiritual support and decreased breathing problems in cancer patients receiving hospice care. Journal of Pain and Symptom Management. 2015; 50(2):225-31 [DOI:10.1016/j.jpainsymman.2015.02.022] [PMID]

[20] Najaf SS, Zareipour H, Yekta Talab SH, Moaref A. [The effect of music therapy on the level of anxiety and vital signs in patients undergoing trans-esophageal echocardiography (Persian)]. Journal of Anesthesiology and Pain. 2018; 9(1):1-11. http://jap.iums.ac.ir/ article-1-5332-fa.htm

[21] do Amaral MA, Neto MG, de Queiroz JG, Martins-Filho PR, Saquetto MB, Oliveira Carvalho V. Effect of music therapy on blood pressure of individuals with hypertension: A systematic review and Meta-analysis. International Journal of Cardiology. 2016; 214:461-4. [DOI:10.1016/j.ijcard.2016.03.197] [PMID]

[22] Liu Y, Petrini MA. Effects of music therapy on pain, anxiety, and vita signs in patients after thoracic surgery. Complementary Therapies in Medicine. 2015; 23(5):714-8. [DOI:10.1016/j.ctim.2015.08.002] [PMID]

[23] Ribeiro AS, Ramos A, Bermejo E, Casero M, Corrales JM, Grantham S. Effects of different musical stimuli in vital signs and facia expressions in patients with cerebral damage: A pilot study. The Journal of Neuroscience Nursing. 2014; 46(2):117-24. [DOI:10.1097/ JNN.0000000000000037] [PMID]

[24] Naderi F, Aghayi A, Mohammadzadeh M, Nazemi S, Salmani $F_{\text {, }}$ Rashvand M. [Comparing the effect of music on pain threshold, anxiety, behavioral responses to pain and the hemodynamic parameters during dressing change in burn patients (Persian)]. Quarterly of the Horizon of Medical Sciences. 2014; 20(1):63-8. https://www.sid.ir/fa/ journal/ViewPaper.aspx?id=238754

[25] Yaghoubinia F, Navidian A, Tabatabaei SM, Sheikh S. [Effect of music on pain intensity in patients with loss of consciousness in intensive care unit (Persian)]. Medical - Surgical Nursing Journal. 2016 4(4):40-7. https://www.sid.ir/fa/journal/ViewPaper.aspx?ID=258109

[26] Çelebi D, Yılmaz E, Şahin ST, Baydur H. The effect of music therapy during colonoscopy on pain, anxiety and patient comfort: A randomized controlled trial. Complementary Therapies in Clinical Practice. 2020; 38:101084. [DOI:10.1016/j.ctcp.2019.101084] [PMID] 
[27] Askary P, Khayat A. [The effectiveness of music therapy on severity of pain, perceived stress and happiness in adolescents with leukemia (Persian)]. Positive Psychology Research. 2018; 3-4(12):15-27. https://ppls.ui.ac.ir/article_22621.html

[28] Jasemi M, Eghtedar S, Aghakhani N, Khodabandeh F, Sayadi L. Music therapy reduces the intensity of pain among patients with cancer. Thrita. 2013; 2(4):76-9. [DOI:10.5812/thrita.13757]

[29] Motahedian E, Movahedirad S, Hajizadeh E, Lak M. The effect of music therapy on postoperative pain intensity in patients under spinal anesthesia. Journal of Critical Care Nursing. 2012; 5(3):139-44. https://www.sid.ir/en/journal/ViewPaper.aspx?id=286136

[30] Luis M, Doss R, Zayed B, Yacoub M. Effect of live oud music on physiological and psychological parameters in patients undergoing cardiac surgery. Global Cardiology Science \& Practice. 2019; (2):e201917. [DOI:10.21542/gcsp.2019.17] [PMID] [PMCID]

[31] Kongsawatvorakul C, Charakorn C, Paiwattananupant K, Lekskul N, Rattanasiri S, Lertkhachonsuk AA. Limited impact of music therapy on patient anxiety with the large loop excision of transformation zone procedure: A randomized controlled trial. Asian Pacific Journal of Cancer Prevention (APJCP). 2016; 17(6):2853-6. [PMID] 